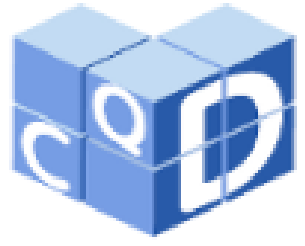

Revista Eletrônica

Paulista de Matemática

ISSN 2316-9664

Volume 21, dez. 2021

Cláudio Estevam Leite da Silva

Universidade Federal de Alfenas/UNIFAL

claudio.estevam@sou.unifalmg.edu.br

\section{Leandro Ferreira}

Universidade Federal de Alfenas/UNIFAL

leandro.ferreira@unifal-

mg.edu.br

\section{Yuri Tavares Pinto}

Universidade Federal de Alfenas/UNIFAL

yuri.pinto@sou.unifal-

mg.edu.br

\section{Aplicativo shiny para o estudo do modelo de risco coletivo com informação fuzzy}

Shiny app for the study of the collective risk model with fuzzy information

\begin{abstract}
Resumo
O presente trabalho tem como objetivo propor um aplicativo interativo desenvolvido em shiny para o estudo do modelo de risco coletivo com informação fuzzy, como apoio ao ensino e pesquisa da modelagem fuzzy em Ciências Atuariais. O aplicativo contempla o modelo de risco coletivo com incertezas presentes nos parâmetros das distribuições de probabilidade, sendo que tais incertezas podem ser modeladas via lógica fuzzy. Em precificação de seguros, o modelo de risco coletivo, que se baseia no estudo do sinistro agregado de uma carteira de apólices, pode ser utilizado no cálculo do prêmio para o segurado. $\mathrm{O}$ aplicativo considera que o parâmetro da distribuição de probabilidade da variável aleatória "número de sinistros ocorridos" é incerto, modelado por um número fuzzy. O aplicativo é uma ferramenta importante para ensino e pesquisa, facilitando a criação de cenários e visualização de resultados mediante alterações nos inputs do usuário.
\end{abstract}

Palavras-chave: Precificação de seguros. Lógica fuzzy. Shiny.

\begin{abstract}
This work aims to propose an interactive application developed in shiny for the study of the collective risk model with fuzzy information, to support the teaching and research of fuzzy modeling in Actuarial Sciences. The application includes the collective risk model with uncertainties present in the parameters of probability distributions, and such uncertainties can be modeled via fuzzy logic. In insurance pricing, the collective risk model, which is based on the study of the aggregate claim of a policy portfolio, can be used to calculate the premium for the insured. The application considers that the parameter of the probability distribution of the random variable "number of claims occurred" is uncertain, modeled by a fuzzy number. The application is an important tool for teaching and research, facilitating the creation of scenarios and visualization of results through changes in user input.
\end{abstract}

Keywords: Insurance pricing. Fuzzy logic. Shiny. 


\section{Introdução}

A indústria de seguros possui inúmeras áreas para aplicações da lógica fuzzy, como na subscrição de seguros, estimativa de mortalidade, classificação de riscos e precificação de seguros (DE ANDRÉS-SÁNCHEZ; GONZÁLEZ-VILA PUCHADES, 2012; KUMAR; JAIN, 2012; PATHAK; DWIVEDI, 2013; SHAPIRO, 2004). Dessa maneira, a lógica fuzzy é uma ferramenta promissora no tratamento de problemas advindos da área de Ciências Atuariais, resultando em uma abordagem alternativa e complementar aos modelos clássicos.

Em precificação de seguros, o modelo de risco coletivo pode ser utilizado no cálculo do prêmio para o segurado, sendo que o prêmio corresponde ao valor que o segurado paga à seguradora pelo seguro. No caso, as seguradoras podem agrupar as apólices em grupos de segurados que apresentam características semelhantes, obtendo diferentes grupos de risco. Informações referentes as características dos segurados, frequência de sinistros e valor do sinistro podem ser consideradas no cálculo do prêmio. No entanto, incertezas presentes nos parâmetros das distribuições de probabilidade, que caracterizam os grupos de riscos, podem ser modeladas via lógica fuzzy, resultando numa abordagem que combina as modelagens fuzzy e estatística. A lógica fuzzy, baseada na teoria dos conjuntos fuzzy, é uma ferramenta utilizada para modelar informações vagas, imprecisas e/ou ambíguas (ZADEH, 1965).

Para analisar o comportamento do modelo de risco coletivo com informação fuzzy, pode-se utilizar o software gratuito $\mathrm{R}$ da área de Computação Estatística, que possui o pacote shiny. Com o pacote shiny, é possível desenvolver aplicações interativas para web (CHANG et al., 2021), permitindo a análise de diferentes cenários de estudo definidos pelo usuário.

O presente trabalho tem como objetivo propor um aplicativo interativo desenvolvido em shiny para o estudo do modelo de risco coletivo com informação fuzzy, como apoio ao ensino e pesquisa da modelagem fuzzy em Ciências Atuariais.

A estrutura deste trabalho apresenta, além desta introdução, no capítulo 2, uma revisão de literatura sobre lógica $f u z z y$, com as definições de conjuntos fuzzy, $\alpha$-nível e números fuzzy. Ainda no capítulo 2, são apresentados o modelo de risco coletivo e o pacote shiny. No capítulo 3, é descrita a metodologia utilizada no trabalho, apresentando o aplicativo desenvolvido e as simulações realizadas. No capítulo 4, são apresentados os resultados das simulações com o uso do aplicativo desenvolvido. No capítulo 5, são apresentadas as considerações finais.

\section{Revisão de literatura}

\subsection{Lógica fuzzy}

\subsubsection{Conjuntos fuzzy}

A lógica fuzzy, também conhecida como lógica nebulosa ou lógica difusa, é uma extensão da lógica clássica e se baseia no tratamento de incertezas via teoria dos conjuntos $f u$ $z z y$. A lógica fuzzy foi inicialmente introduzida por Zadeh (1965), tendo como objetivo, trabalhar informações imprecisas.

Um conjunto clássico pode ser caracterizado por sua função característica. De acordo com Barros, Bassanezi e Lodwick (2017), sejam $U$ um conjunto universo e $A$ um subconjunto de $U$, a função característica de $A$ é definida como 


$$
x_{A}(x)=\left\{\begin{array}{lll}
1 & \text { se } x & \in A \\
0 & \text { se } x & \notin
\end{array} .\right.
$$

Assim, $x_{A}$ é uma função cujo domínio é $U$ e imagem contida no conjunto $\{0,1\}$, ou seja, $x_{A}(x)=1$ significa que $x$ pertence ao subconjunto $A$, e se $x_{A}(x)=0, x$ não é elemento de $A$. Um conjunto ou subconjunto fuzzy $A$ de um conjunto universo $U$ é definido por uma função de pertinência $\mu_{A}$, representada por

$$
\mu_{A}(x): U \rightarrow[0,1]
$$

O valor obtido para $\mu_{A}(x) \in[0,1]$ indica o grau de pertinência com que o elemento $x$ de $U$ pertence ao conjunto fuzzy $A$.

\subsection{2 $\alpha$-nível}

Conforme Barros, Bassanezi e Lodwick (2017), para todo subconjunto fuzzy é possível associar uma série de conjuntos clássicos denominados de $\alpha$-níveis de $A$. Um elemento $x$ do subconjunto $A$ pertence a determinado $\alpha$-nível se seu grau de pertinência é maior que um determinado valor $\alpha \in[0,1]$. Este conjunto clássico é denotado por $[A]^{\alpha}$, sendo

$$
[A]^{\alpha}=\left\{x \in U: \mu_{A}(x) \geq \alpha\right\}, \text { para } 0<\alpha \leq 1 .
$$

Define-se $[A]^{0}$ como o fecho do suporte de $A$, que é indicado por

$$
\overline{\operatorname{supp}} A=\overline{\left\{x \in U: \mu_{A}(x)>0\right\}}
$$

\subsubsection{Números fuzzy}

Para Barros, Bassanezi e Lodwick (2017), um subconjunto fuzzy $A$ é considerado um número fuzzy quando o conjunto universo de $\mu_{A}(x)$ é o conjunto dos números reais $(\mathbb{R})$ e satisfaz as seguintes condições:

i) todos os $\alpha$-níveis de $A$ são intervalos fechados e não vazios de $\mathbb{R}$;

ii) o conjunto $\left\{x \in U: \mu_{A}(x)>0\right\}$ é um conjunto limitado de $\mathbb{R}$.

Os $\alpha$-níveis de um número fuzzy $A$ podem ser denotados por $[A]^{\alpha}=\left[a_{1}^{\alpha}, a_{2}^{\alpha}\right]$, para todo $\alpha \in[0,1]$.

Os números fuzzy mais comuns são os triangulares, trapezoidais e em forma de sino.

Seja $A$ um número fuzzy triangular, sua função de pertinência é

$$
\mu_{A}(x)=\left\{\begin{array}{l}
\frac{x-a}{b-a}, \text { se } a<x \leq b ; \\
\frac{c-x}{c-b}, \text { se } b<x \leq c ; \\
0, \text { caso contrário. }
\end{array}\right.
$$

Tendo como base o intervalo da reta $[a, c]$ e $\mu_{A}(b)=1$, o número fuzzy triangular é definido por $A=(a ; b ; c)$ e seus $\alpha$-níveis têm a forma

$$
\left[a_{1}^{\alpha}, a_{2}^{\alpha}\right]=[(b-a) \alpha+a,-(c-b) \alpha+c],
$$


para todo $\alpha \in[0,1]$.

Seja $A$ um número fuzzy trapezoidal, sua função de pertinência é

$$
\mu_{A}(x)=\left\{\begin{array}{l}
\frac{x-a}{b-a}, \text { se } a \leq x<b \\
1, \text { se } b \leq x \leq c \\
\frac{d-x}{d-c}, \text { se } c<x \leq d \\
0, \text { caso contrário. }
\end{array}\right.
$$

Os $\alpha$-níveis de um número fuzzy trapezoidal são dados por

$$
\left[a_{1}^{\alpha}, a_{2}^{\alpha}\right]=[(b-a) \alpha+a,-(d-c) \alpha+d]
$$

para todo $\alpha \in[0,1]$.

As operações aritméticas, considerando números fuzzy, estão relacionadas com as operações aritméticas intervalares. Barros, Bassanezi e Lodwick (2017) apresentam operações intervalares com números fuzzy.

Sejam $\lambda$ um número real e, $A$ e $B$ números fuzzy com $\alpha$-níveis dados por $[A]^{\alpha}=$ $\left[a_{1}^{\alpha}, a_{2}^{\alpha}\right]$ e $[B]^{\alpha}=\left[b_{1}^{\alpha}, b_{2}^{\alpha}\right]$, têm-se as seguintes propriedades:

a) A soma entre $A$ e $B$ é o número fuzzy $A+B$, com $\alpha$-níveis

$$
[A+B]^{\alpha}=[A]^{\alpha}+[B]^{\alpha}=\left[a_{1}^{\alpha}+b_{1}^{\alpha}, a_{2}^{\alpha}+b_{2}^{\alpha}\right] .
$$

b) A diferença entre $A$ e $B$ é o número fuzzy $A-B$, com $\alpha$-níveis

$$
[A-B]^{\alpha}=[A]^{\alpha}-[B]^{\alpha}=\left[a_{1}^{\alpha}-b_{2}^{\alpha}, a_{2}^{\alpha}-b_{1}^{\alpha}\right] .
$$

c) A multiplicação de $A$ por um escalar $\lambda$ é o número fuzzy $\lambda A$, $\operatorname{com} \alpha$-níveis

$$
[\lambda A]^{\alpha}=\lambda[A]^{\alpha}= \begin{cases}{\left[\lambda a_{1}^{\alpha}, \lambda a_{2}^{\alpha}\right],} & \text { se } \lambda \geq 0 \\ {\left[\lambda a_{2}^{\alpha}, \lambda a_{1}^{\alpha}\right],} & \text { se } \lambda<0\end{cases}
$$

d) A multiplicação de $A$ por $B$ é o número fuzzy $A \cdot B, \operatorname{com} \alpha$-níveis

$$
[A \cdot B]^{\alpha}=[A]^{\alpha} \cdot[B]^{\alpha}=[\min P, \max P],
$$

em que $P=\left\{a_{1}^{\alpha} b_{1}^{\alpha}, a_{1}^{\alpha} b_{2}^{\alpha}, a_{2}^{\alpha} b_{1}^{\alpha}, a_{2}^{\alpha} b_{2}^{\alpha}\right\}$.

e) A divisão de $A$ por $B$, se $0 \notin \operatorname{supp} B$, é o número $f u z z y \frac{A}{B}$, com $\alpha$-níveis

$$
\left[\frac{A}{B}\right]^{\alpha}=\frac{[A]^{\alpha}}{[B]^{\alpha}}=\left[a_{1}^{\alpha}, a_{2}^{\alpha}\right] \cdot\left[\frac{1}{b_{2}^{\alpha}}, \frac{1}{b_{1}^{\alpha}}\right] .
$$




\subsection{Modelo de risco coletivo}

O modelo clássico de risco coletivo de seguros teve sua origem com a publicação da tese do matemático sueco Filip Oskar Lundberg, no ano de 1903, intitulado Approximations of the Probability Function Reinsurance of Collective Risks. Lundberg utilizou um processo de Poisson homogêneo para modelar as indenizações que chegavam a uma seguradora até um tempo $t$. Posteriormente, em 1930, Harald Cramér deu continuidade ao estudo de Lundberg, surgindo o modelo clássico de Cramér-Lundberg ou modelo clássico de risco coletivo. O modelo clássico de Cramér-Lundberg é um processo estocástico que calcula a reserva da seguradora até o instante $t$, levando em consideração, as indenizações agregadas (sinistro agregado) no intervalo $(0, t]$, a reserva inicial da seguradora e os prêmios recebidos a uma taxa constante por unidade de tempo (SHIRYAEV, 1999).

$\mathrm{Na}$ teoria do risco coletivo, pode-se analisar o sinistro agregado $S$ de uma seguradora, assumindo que $S$ é uma variável aleatória que segue uma distribuição composta (TSE, 2009). Assim, seja $N$ a variável aleatória "número de sinistros ocorridos" e seja $X_{i}$ a variável aleatória "valor do sinistro referente a $i$-ésima ocorrência" (sinistro individual), então o sinistro agregado $S$ será dado por:

$$
S=X_{1}+X_{2}+\cdots+X_{N}=\sum_{i=1}^{N} X_{i}
$$

Neste modelo, assume-se que $X_{1}, X_{2}, \ldots, X_{N}$ sejam independentes e identicamente distribuídas e que $N$ e $X$ são independentes. A seguir, são mencionados alguns modelos probabilísticos e aproximações de distribuições que podem ser aplicados no estudo de $N, X$ e $S$, conforme Pacheco (2014):

i) Modelos probabilísticos para $N$ : Binomial, Poisson e Binomial Negativa;

ii) Modelos probabilísticos para $X$ : Aritmética, Gama Generalizada, LogGama, Beta Generalizada, Normal, LogNormal, Benktander e Generalizada de Valores Extremos;

iii) Aproximações de distribuições para $S$ : Normal, Gama, Edgeworth, Normal Power e simulações de Monte Carlo.

Com a obtenção do sinistro agregado $S$, o prêmio agregado $P$ pode ser encontrado, sendo que $P$ deverá ser suficiente para cobrir o sinistro agregado esperado $(E(S))$, com certo carregamento de segurança $(C S)$. Existem diferente princípios de cálculos de prêmios que devem satisfazer propriedades desejáveis, como a propriedade de positividade de carregamento, onde o prêmio agregado deve conter um carregamento positivo em relação ao valor esperado do sinistro agregado, ou seja, $P>E(S)$ (PACHECO, 2014).

\subsection{Pacote shiny}

Shiny é um pacote no ambiente R, que é um software livre e gratuito da área de Computação Estatística (THE R PROJECT FOR STATISTICAL COMPUTING, 2021). Com o pacote shiny é possível desenvolver aplicações interativas para web (CHANG et al., 2021), onde o usuário pode analisar diferentes cenários de estudo, modificando as informações de entrada (inputs) através de uma interface e obtendo resultados (outputs) de maneira direita.

A estrutura de programação no pacote shiny é composta pelos componentes UI (user interface) e Server. O componente UI é responsável por construir o layout do aplicativo e o 
componente Server é responsável pelas análises que são executadas pelo software R. A aplicação interativa pode ser acessada de forma online, por um navegador web.

\section{Metodologia}

A página inicial do aplicativo interativo desenvolvido em shiny (Figura 1), chamado fuzzyLAR, contém as seguintes abas:

i) UNIFAL-MG: ao clicar, o usuário é direcionado para o site da Universidade Federal de Alfenas (UNIFAL);

ii) Introdução: apresenta informações sobre o aplicativo;

iii) Lógica fuzzy: apresenta uma breve explicação sobre lógica fuzzy; lação 2",

iv) Modelo de risco coletivo: apresenta as subabas "Tutorial", "Simulação 1" e "Simu-

v) Referências: apresenta as referências bibliográficas utilizadas na elaboração das análises.

A página inicial do aplicativo contém os logos do Laboratório de Risco Atuarial e Inovação (LAR - UNIFAL) e do grupo de pesquisa do CNPq Núcleo de Estudos em Matemática Fuzzy (NEMAF - UNIFAL).

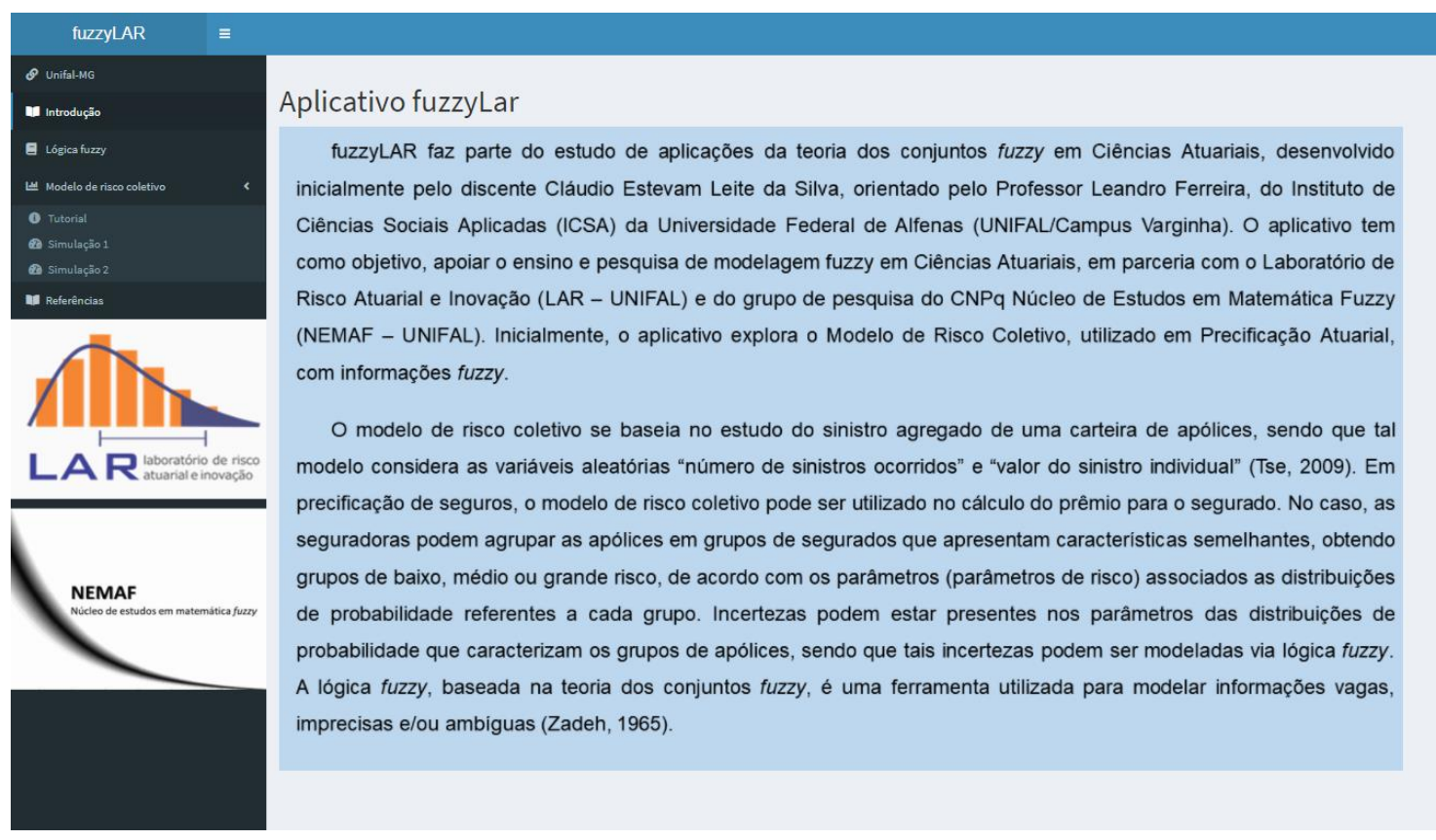

Figura 1 - Página inicial do aplicativo fuzzyLAR.

A seguir, serão apresentadas as simulações que estão presentes nas subabas "Simulação 1" e "Simulação 2". A Simulação 1 consiste na simulação de uma variável aleatória contínua, considerando que o parâmetro da distribuição é incerto, representado por um número fuzzy. A Simulação 2 consiste na análise do comportamento do modelo de risco coletivo, considerando que o parâmetro da distribuição da variável aleatória número de sinistros ocorridos é incerto, representado por um número fuzzy. 


\subsection{Simulação 1 - Distribuição exponencial com informação fuzzy}

Foi realizada a simulação de uma variável aleatória $X$ que segue uma distribuição exponencial, em que o parâmetro $\theta$ desta distribuição é o número fuzzy triangular $\tilde{\theta}=(a ; b ; c)$. Assim, foi observado o comportamento desta distribuição para diferentes $\alpha$-níveis do número fuzzy triangular $\tilde{\theta}$.

Considerando $X$ uma variável aleatória com distribuição exponencial, ou seja, $X \sim \operatorname{Exponencial}(\theta)$, sabe-se que a função de distribuição acumulada de $X$ é:

$$
F_{X}(x)=1-e^{-\theta x}
$$

Pelo método da transformada inversa (RIZZO, 2007), tem-se que:

$$
\begin{aligned}
& F_{X}(x)=u=1-e^{-\theta x} \\
& e^{-\theta x}=(1-u) \\
& -\theta x=\ln (1-u) \\
& x=\frac{-\ln (1-u)}{\theta}
\end{aligned}
$$

Para uma sequência de valores de $u$, sendo que $u \in[0,1]$, foram encontrados os valores correspondentes de $X$. Após obtidos os valores de $X$, pôde-se observar o comportamento da função de distribuição acumulada para diferentes $\alpha$-níveis de $\tilde{\theta}$, aqui representados por $\tilde{\theta}^{\alpha}=\left[\tilde{\theta}_{1}^{\alpha}, \tilde{\theta}_{2}^{\alpha}\right]=\left[\tilde{\theta}_{\text {inf }}^{\alpha}, \tilde{\theta}_{\text {sup }}^{\alpha}\right]$, em que $\tilde{\theta}_{\text {inf }}^{\alpha}=(b-a) \alpha+a$ representa o limite inferior e $\tilde{\theta}_{\text {sup }}^{\alpha}==-(c-b) \alpha+c$ representa o limite superior de $\tilde{\theta}^{\alpha}$. Assim, com base em tais limites, foram observados os comportamentos das funções de distribuição acumuladas $F_{X}(x)_{\text {inf }}^{\alpha} \mathrm{e}$ $F_{X}(x)_{\text {sup }}^{\alpha}$. A seguir, é apresentado o algoritmo para obter $F_{X}(x)_{i n f}^{\alpha}$ e $F_{X}(x)_{\text {sup }}^{\alpha}$.

Algoritmo para obter $\boldsymbol{F}_{\boldsymbol{X}}(\boldsymbol{x})_{\text {inf }}^{\alpha}$ e $\boldsymbol{F}_{\boldsymbol{X}}(\boldsymbol{x})_{\text {sup }}^{\alpha}$ :

1. Definir um número fuzzy triangular $\tilde{\theta}=(a ; b ; c)$;

2. Definir uma sequência de valores de $u \in[0,1]$;

3. Encontrar uma sequência de valores de $x$, sendo

$$
x=\frac{-\ln (1-u)}{b}
$$

4. Escolher o valor de $\alpha$;

5. Encontrar $\tilde{\theta}_{\text {inf }}^{\alpha}=(b-a) \alpha+a$;

6. Encontrar $\tilde{\theta}_{\text {sup }}^{\alpha}==-(c-b) \alpha+c$;

7. Para cada valor de $x$, obter $F_{X}(x)_{i n f}^{\alpha}=1-e^{-\widetilde{\theta}_{i n f}^{\alpha} x}$;

8. Para cada valor de $x$, obter $F_{X}(x)_{\text {sup }}^{\alpha}=1-e^{-\widetilde{\theta}_{\text {sup }}^{\alpha} x}$.

\subsection{Simulação 2 - Modelo de risco coletivo com informação fuzzy}


Foi realizado o estudo do modelo de risco coletivo (TSE, 2009), considerando $N$ como o número de sinistros ocorridos e $X_{i}$ como o valor do sinistro referente a $i$-ésima ocorrência. O sinistro agregado $S$ é dado por

$$
S=\sum_{i=1}^{N} X_{i} .
$$

Primeiramente, obteve-se um valor para a variável aleatória $N$ de acordo com uma distribuição de Poisson com parâmetro $\lambda$. O parâmetro $\lambda$ desta distribuição foi considerado como um número fuzzy triangular $\tilde{\lambda}=(a ; b ; c)$. Com base nos valores simulados de $N$, foram obti$\operatorname{dos} N$ valores de $X$ que seguem uma distribuição gama com parâmetros $\gamma$ e $\beta$, ou seja, $X \sim \operatorname{Gama}(\gamma, \beta)$, com $E(X)=\gamma / \beta$. Considerando os diferentes $\alpha$-níveis de $\tilde{\lambda}$, aqui representados por $[\tilde{\lambda}]^{\alpha}=\left[\lambda_{1}^{\alpha}, \lambda_{2}^{\alpha}\right]=\left[\tilde{\lambda}_{\text {inf }}^{\alpha}, \tilde{\lambda}_{\text {sup }}^{\alpha}\right]$, em que $\tilde{\lambda}_{\text {inf }}^{\alpha}=(b-a) \alpha+a$ e $\tilde{\lambda}_{\text {sup }}^{\alpha}=$ $-(c-b) \alpha+c$, foram observados os comportamentos dos sinistros agregados $S_{\text {inf }}^{\alpha}$ e $S_{\text {sup }}^{\alpha}$. A seguir, são apresentados os algoritmos para obter amostras de $S_{\text {inf }}^{\alpha}$ e $S_{\text {sup }}^{\alpha}$ para um $\alpha$-nível específico, a partir da escolha inicial dos valores de $a, b, c, \gamma$ e $\beta$.

\section{Algoritmo para obter uma amostra de $S_{\text {inf }}^{\alpha}$ :}

1. Simular um valor de $N$, sabendo que $N \sim \operatorname{Poisson}\left(\tilde{\lambda}_{\text {inf }}^{\alpha}\right)$;

2. Simular $N$ valores de $X$, sabendo que $X \sim \operatorname{Gama}(\gamma, \beta)$;

3. Encontrar

$$
S_{i n f}^{\alpha}=\sum_{i=1}^{N} X_{i}
$$

4. Repetir os passos anteriores $M$ vezes para obter uma amostra de $S_{i n f}^{\alpha}$.

\section{Algoritmo para obter uma amostra de $S_{\text {sup }}^{\alpha}$ :}

1. Simular um valor de $N$, sabendo que $N \sim \operatorname{Poisson}\left(\tilde{\lambda}_{\text {sup }}^{\alpha}\right)$;

2. Simular $N$ valores de $X$, sabendo que $X \sim \operatorname{Gama}(\gamma, \beta)$;

3. Encontrar

$$
S_{\text {sup }}^{\alpha}=\sum_{i=1}^{N} X_{i}
$$

4. Repetir os passos anteriores $M$ vezes para obter uma amostra de $S_{\text {sup }}^{\alpha}$.

Encontradas $M$ simulações de $S_{\text {inf }}^{\alpha}$ e $S_{\text {sup }}^{\alpha}$, foi obtido o valor do prêmio $(P)$ pelo Princípio do Valor Esperado (PACHECO, 2014), dado por:

$$
P=(1+C S) E(S)
$$

em que $C S$ é o carregamento de segurança $(C S>0)$ e $E(S)$ é o valor esperado do sinistro agregado $S$. Considerando um determinado valor para $C S$ e as amostras de $S_{\text {inf }}^{\alpha}$ e $S_{\text {sup }}^{\alpha}$, foram obtidos os prêmios $P_{\text {inf }}^{\alpha}=(1+C S) E\left(S_{\text {inf }}^{\alpha}\right)$ e $P_{\text {sup }}^{\alpha}=(1+C S) E\left(S_{\text {sup }}^{\alpha}\right)$. 


\section{Resultados e discussão}

A seguir, serão apresentados cenários referentes as simulações 1 e 2 para exemplificar o uso do aplicativo.

\subsection{Simulação 1 - Distribuição exponencial com informação fuzzy}

Considerando o número fuzzy triangular $\tilde{\theta}=(0,4 ; 0,5 ; 0,6)$ para o parâmetro da distribuição exponencial, para $\alpha=1,0$, tem-se o $\alpha$-nível com pertinência máxima, em que os limites $\tilde{\theta}_{\text {inf }}^{1,0}$ e $\tilde{\theta}_{\text {sup }}^{1,0}$ são iguais, assim como as funções de distribuição acumuladas $F_{X}(x)_{\text {inf }}^{1,0}$ e $F_{X}(x)_{\text {sup }}^{1,0}$. Dessa maneira, para $\alpha=1,0$, tem-se o caso clássico, sendo que $\theta_{\text {inf }}^{1,0}=\theta_{\text {sup }}^{1,0}=\mu=$ 0,5 . Para $x=4$ e $\alpha=1,0$, tem-se que $F_{X}(4)_{\text {inf }}^{1,0}=F_{X}(4)_{\text {sup }}^{1,0}=0,86$. Para um nível de incerteza igual a $0,2(\alpha=0,2)$, tem-se que $\tilde{\theta}_{\text {inf }}^{0,2}=0,42$ e $\tilde{\theta}_{\text {sup }}^{0,2}=0,58$, sendo que para $x=4$, tem-se que $F_{X}(4)_{\text {inf }}^{0,2}=0,81$ e $F_{X}(4)_{\text {sup }}^{0,2}=0,90$. É possível notar que a maior amplitude dos valores das funções de distribuição acumuladas ocorre com a redução do $\alpha$-nível, ou seja, quanto maior a incerteza sobre $\theta$, maior será esta amplitude.

A Figura 2 apresenta a tela que traz o gráfico da função de pertinência gerado pelo aplicativo referente ao número fuzzy triangular $\tilde{\theta}=(0,4 ; 0,5 ; 0,6)$, definindo $a=0,4, b=$ 0,5 e $c=0,6$.

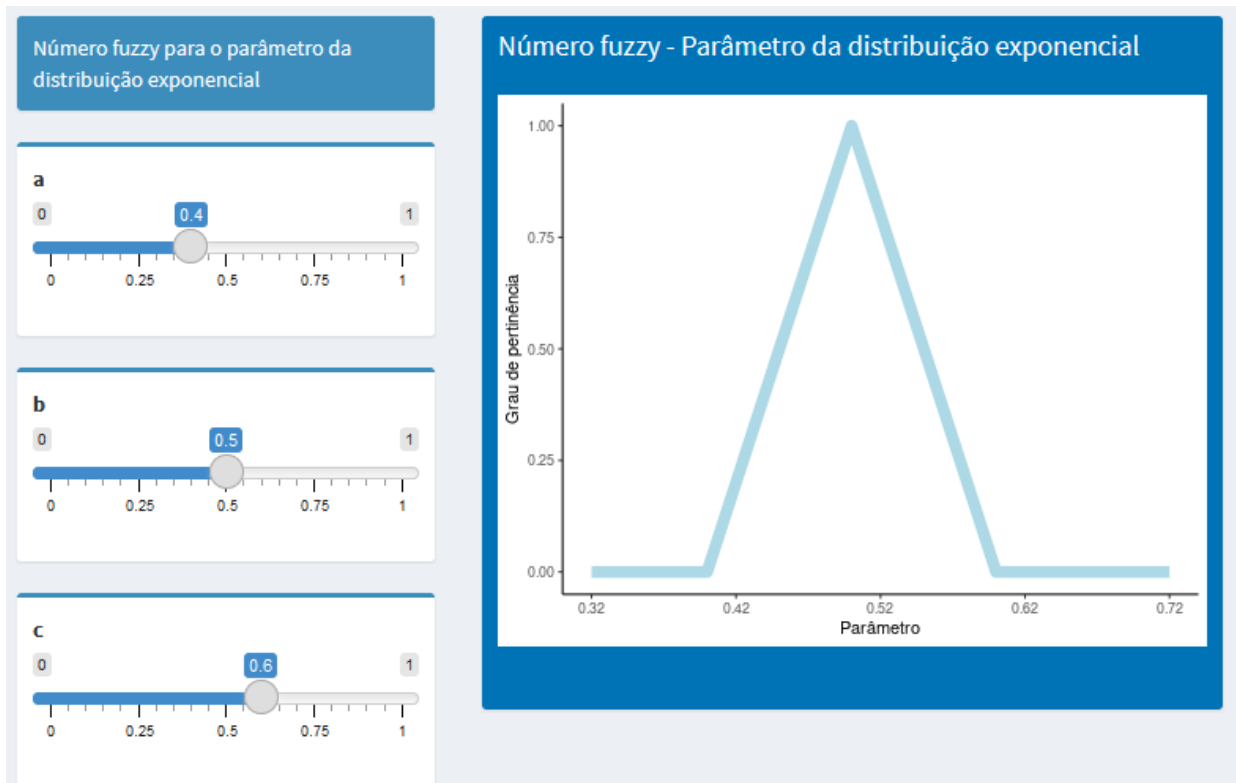

Figura 2 - Gráfico da função de pertinência de $\tilde{\theta}=(0,4 ; 0,5 ; 0,6)$.

Considerando o número fuzzy $\tilde{\theta}=(0,4 ; 0,5 ; 0,6)$, a Figura 3 traz as funções de distribuição acumuladas para um nível de incerteza igual a 0,8 , ou seja, $\alpha=0,8$, geradas pelo aplicativo. As curvas em azul e vermelho representam os gráficos de $F_{X}(x)_{\text {sup }}^{0,8}$ e $F_{X}(x)_{\text {inf }}^{0,8}$, respectivamente. Na barra "Nível", o usuário pode escolher o nível de incerteza. A Figura 4 apresenta a tela completa referente a Simulação 1. 


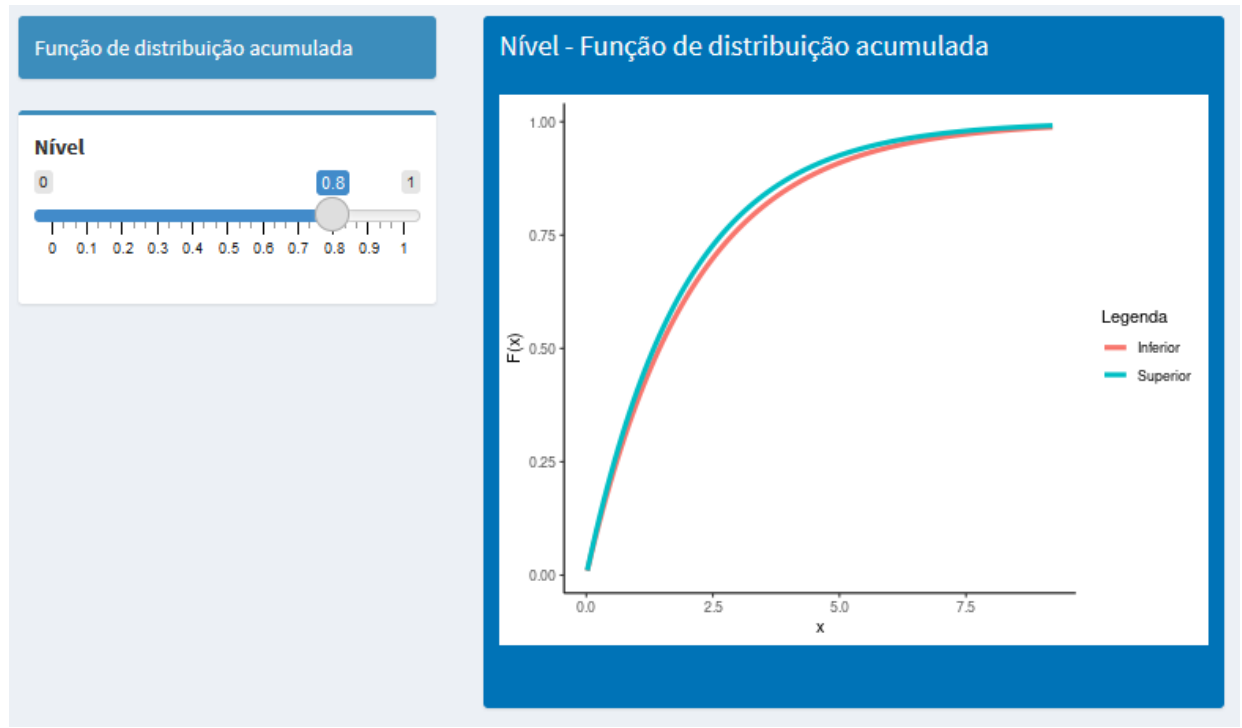

Figura 3 - Gráficos das funções de distribuição acumuladas para $\alpha=0,8$.

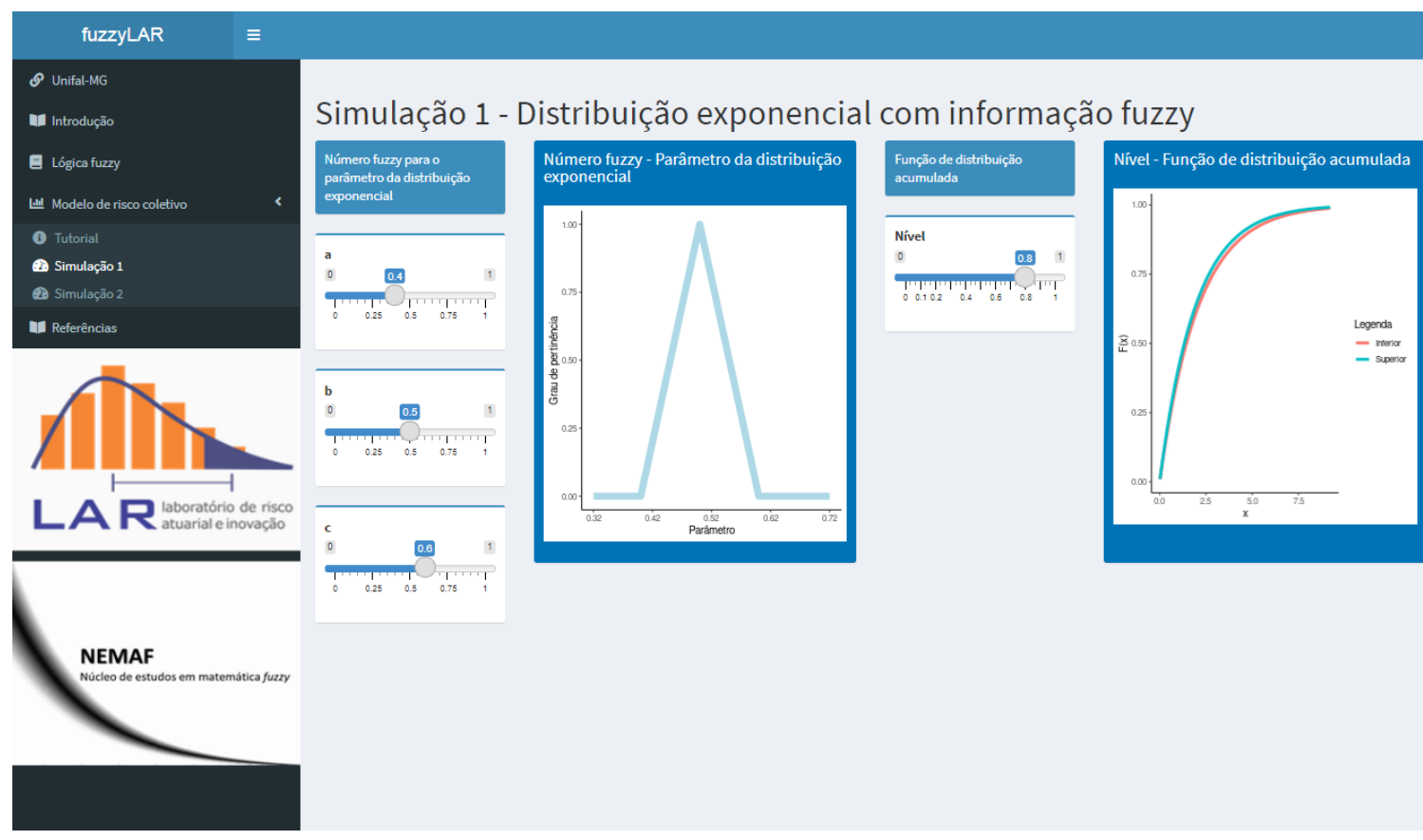

Figura 4 - Tela Simulação 1.

\subsection{Simulação 2 - Modelo de risco coletivo com informação fuzzy}

Para o modelo de risco coletivo com informação fuzzy, foi considerada que a variável aleatória $N$ segue uma distribuição de Poisson com parâmetro $\lambda$, sendo que $\lambda$ é representado pelo número fuzzy triangular $\tilde{\lambda}=(4 ; 5 ; 6)$. Também foi considerado que $X$ segue uma distribuição gama com parâmetros $\gamma=100$ e $\beta=0,02$.

De acordo com diferentes valores de $\alpha$-níveis e $\tilde{\lambda}^{\alpha}=\left[\lambda_{\text {inf }}^{\alpha}, \lambda_{\text {sup }}^{\alpha}\right]$, a Tabela 1 apresenta os resultados obtidos para 1000 simulações, onde M1 e M2 representam as médias referen- 
tes a $S_{\text {inf }}^{\alpha}$ e $S_{\text {sup }}^{\alpha}$, respectivamente. Na Tabela 1, também são apresentadas as diferenças (DIF) entre M2 e M1. Pode-se notar que as DIFs diminuem com a redução do valor do $\alpha$-nível, ou seja, quanto menor a incerteza sobre $\lambda$, menor a diferença entre M2 e M1.

Tabela 1 - Resultados obtidos referentes as 1000 simulações de $S_{\text {inf }}^{\alpha}$ e $S_{\text {sup }}^{\alpha}$.

\begin{tabular}{cccccc}
\hline$\alpha$ - nível & $\lambda_{\text {inf }}^{\alpha}$ & $\lambda_{\text {sup }}^{\alpha}$ & M1 & M2 & DIF \\
\hline 0,1 & 4,1 & 5,9 & 20383,50 & 29728,36 & 9344,86 \\
0,3 & 4,3 & 5,7 & 21473,10 & 28529,45 & 7056,35 \\
0,7 & 4,7 & 5,3 & 23400,69 & 26606,31 & 3205,62 \\
\hline
\end{tabular}

Fonte: Elaboração própria.

A Figura 5 apresenta a tela que traz o gráfico da função de pertinência gerado pelo aplicativo referente ao número fuzzy triangular $\tilde{\lambda}=(4 ; 5 ; 6)$, definindo $a=4, b=5$ e $c=6$. Para visualizar o comportamento da distribuição de $X$, que segue uma distribuição gama com parâmetros $\gamma=100$ (parâmetro de forma) e $\beta=0,02$ (parâmetro de escala), o aplicativo apresenta o histograma de valores simulados de $X$ (Figura 6). Considerando 1000 simulações, a Figura 6 apresenta os histogramas de $S_{\text {inf }}^{\alpha}$ (em vermelho) e $S_{\text {sup }}^{\alpha}$ (em azul) gerados pelo aplicativo, para um nível de incerteza igual a $0,8(\alpha=0,8)$. Na tela apresentada na Figura 7 , o usuário pode escolher o número de simulações e o nível de incerteza a serem adotados.

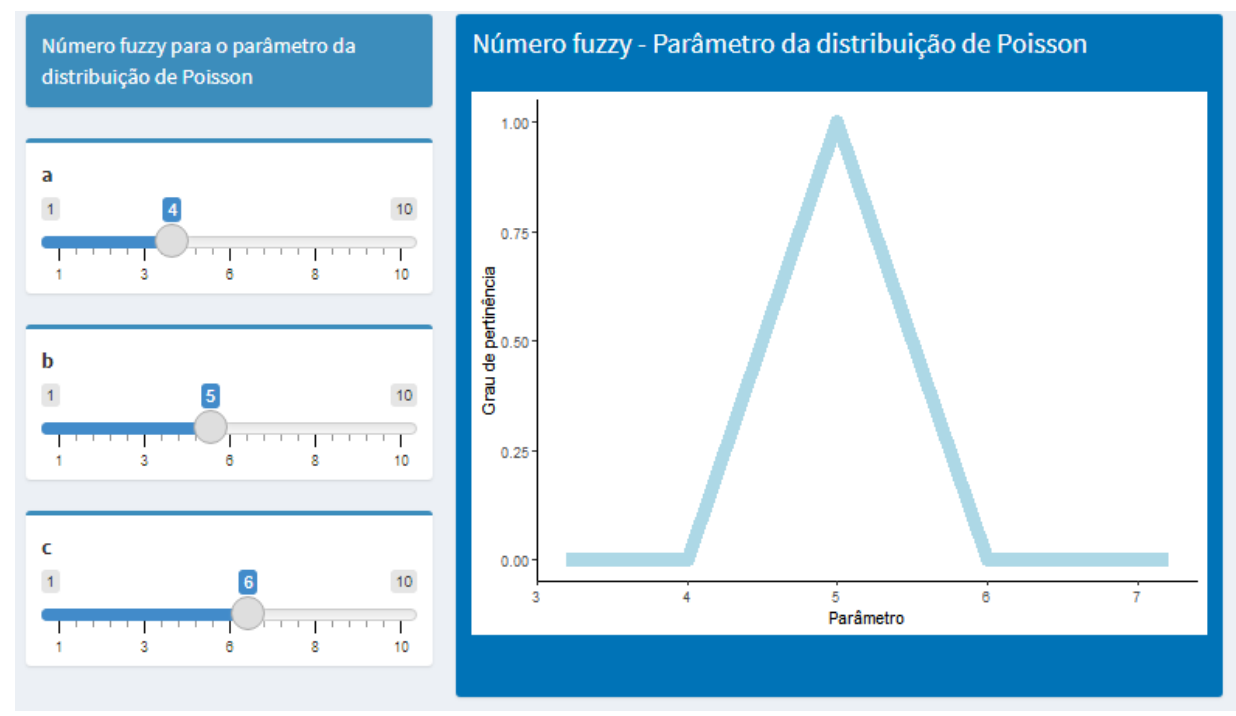

Figura 5 - Gráfico da função de pertinência de $\tilde{\lambda}=(4 ; 5 ; 6)$. 


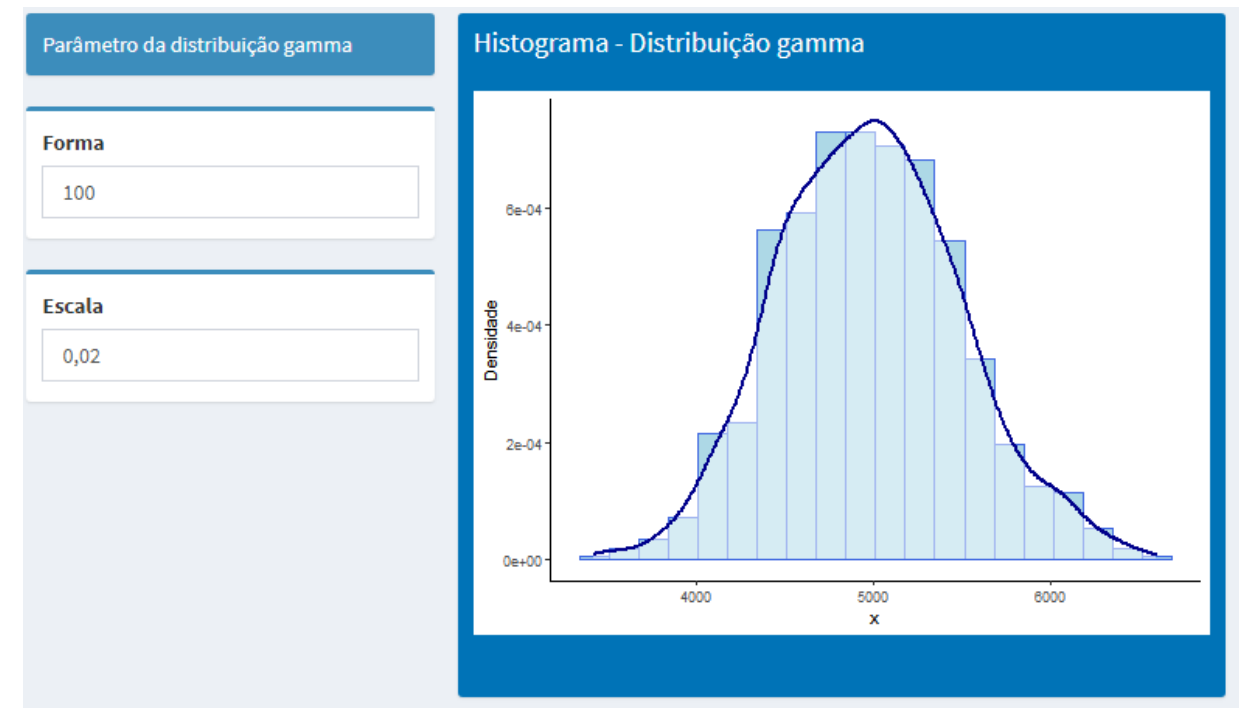

Figura 6 - Histograma da distribuição de $X$.

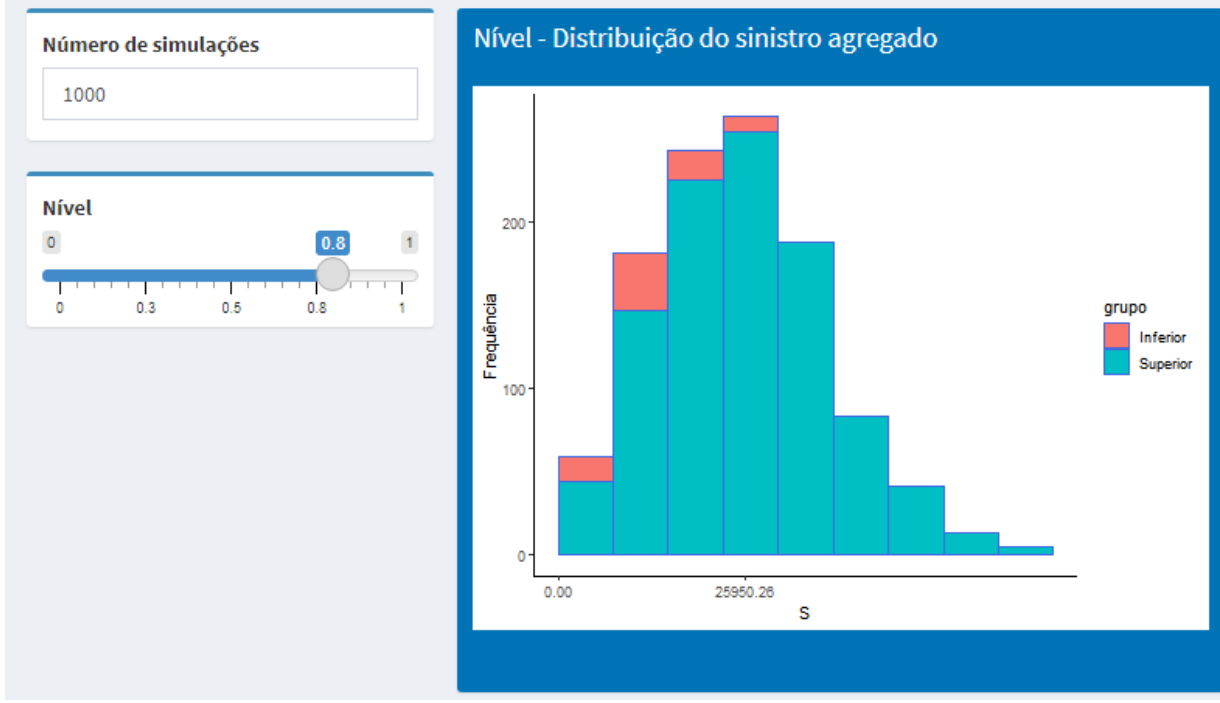

Figura 7 - Histogramas de $S_{\text {inf }}^{\alpha}$ e $S_{\text {sup }}^{\alpha}$ para $\alpha=0,8$.

Por fim, foi encontrado o valor do prêmio agregado com base no Princípio do Valor Esperado, considerando as 1000 simulações de $S_{\text {inf }}^{\alpha}$ e $S_{\text {sup }}^{\alpha}$, para $\alpha=0,8$. Para tanto, foi escolhido um carregamento de segurança igual a $0,8(C S=0,8)$. A Figura 8 apresenta a tela gerada pelo aplicativo com os valores obtidos para $P_{\text {inf }}^{\alpha}$ (Valor do prêmio inferior) e $P_{\text {sup }}^{\alpha}$ (Valor do prêmio superior). A Figura 9 apresenta a tela completa referente a Simulação 2. 


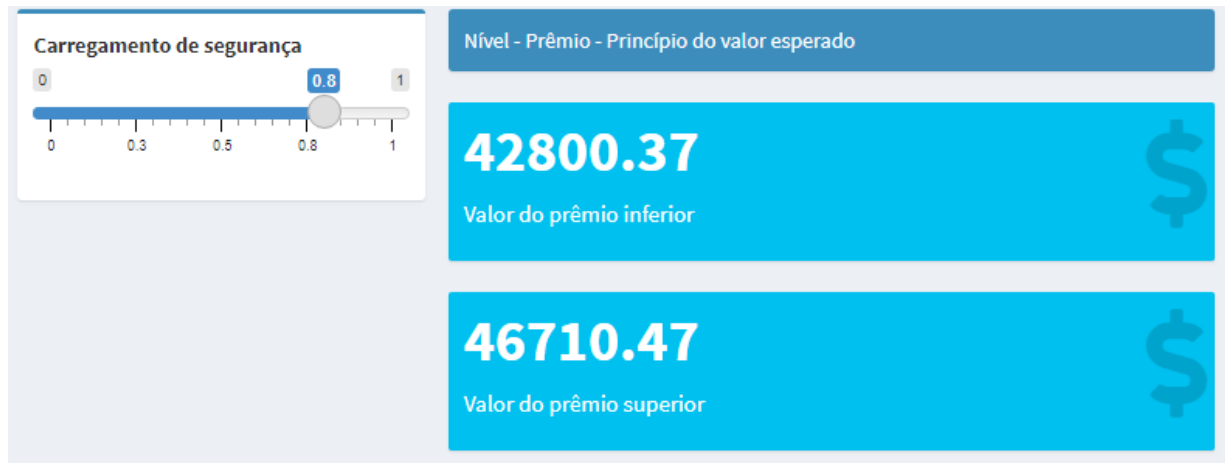

Figura 8 - Valores dos prêmios para $C S=0,8$.

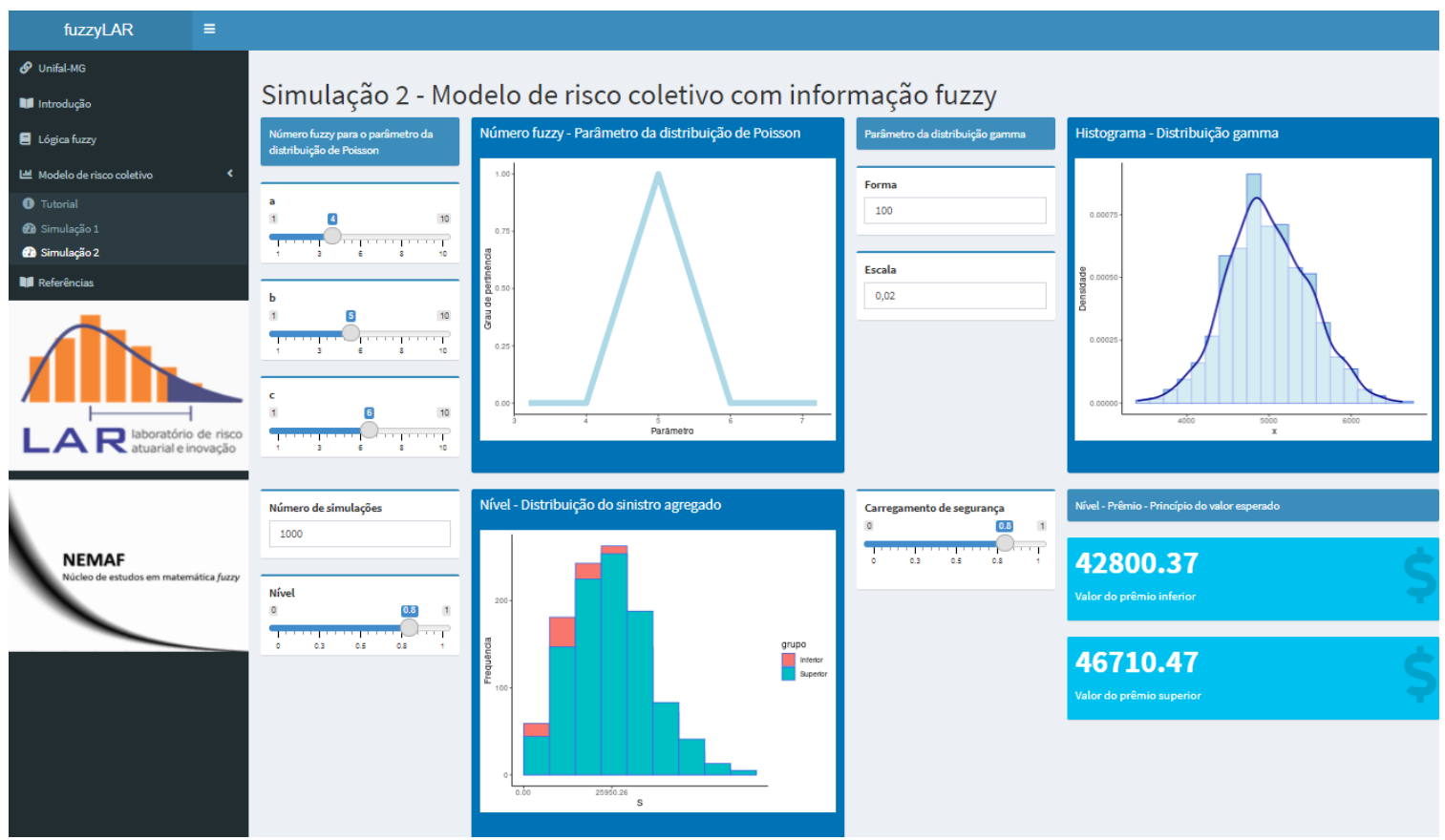

Figura 9 - Tela Simulação 2.

\section{Considerações finais}

O presente trabalho teve como objetivo propor um aplicativo interativo desenvolvido em shiny para o estudo do modelo de risco coletivo com informação fuzzy, como apoio ao ensino e pesquisa da modelagem fuzzy em Ciências Atuariais. A proposta é uma alternativa viável para o tratamento de incertezas presentes no modelo de risco coletivo, que é utilizado no cálculo do prêmio para o segurado, resultando num estudo mais completo devido a níveis de incertezas que podem ser adotados. Em breve, o aplicativo estará disponível na web, com livre acesso.

A aplicação da lógica fuzzy possibilita diferentes interpretações e perspectivas do modelo de risco coletivo, permitindo a avaliação de diferentes estratégias no momento da precificação, buscando maior segurança financeira para a seguradora, ou até mesmo oferecendo produtos mais atrativos para o segurado.

Para trabalhos futuros, pretende-se ampliar as análises disponíveis no aplicativo, abordando, como por exemplo, sistemas baseados em regras fuzzy e regressão fuzzy. 


\section{Referências}

BARROS, L. C.; BASSANEZI, R. C.; LODWICK, W. A. First course in fuzzy logic, fuzzy dynamical systems, and biomathematics. Berlin: Springer-Verlag, 2017.

CHANG, W. et al. Shiny: web application framework for R. [S.l:s.n], 2021. Disponível em: https://CRAN.R-project.org/package=shiny. Acesso em: 10 mai. 2021.

DE ANDRÉS-SÁNCHEZ, J.; GONZÁLEZ-VILA PUCHADES, L. Using fuzzy random variables in life annuities pricing. Fuzzy sets and systems, v. 188, n. 1, p. 27-44, fev. 2012.

KUMAR, S.; JAIN, H. A fuzzy logic based model for life insurance underwriting when insured is diabetic. European Journal of Applied Sciences, v. 4, n. 5, p. 196-202, jan. 2012.

PACHECO, R. Matemática atuarial de seguros de danos. São Paulo: Atlas, 2014.

PATHAK, P.; DWIVEDI, V. K. Fuzzy based pricing model for old age insurance.

International Journal of Fuzzy Logic Systems, v. 3, n. 1, p. 47-54, jan. 2013.

RIZZO, M. L. Statistical computing with R. Boca Raton: Chapman and Hall/CRC, 2007.

SHAPIRO, A. F. Fuzzy logic in insurance. Insurance: Mathematics and Economics, v. 35, n. 2, p. 399-424, out. 2004.

SHIRYAEV, A. N. Essentials of stochastic finance: facts, models, theory. Singapore: World Scientific, 1999.

THE R project for statistical computing. [S.l.]: The R Foundation, 2021. Disponível em: https://www.r-project.org/. Acesso em: 14 set. 2021.

TSE, Y. K. Nonlife actuarial models: theory, methods and evaluation. Cambridge: Cambridge University Press, 2009.

ZADEH, L. A. Fuzzy sets. Information and Control, v. 8, n. 3, p. 338-353, jun.1965. 